\title{
Investigation of the nucleon-nucleon tensor force in the three-nucleon system
}

\author{
M. Clajus ${ }^{\text {a }}$, P.M. Egun ${ }^{\text {a }}$, W. Grüebler ${ }^{\text {a }}$, P. Hautle ${ }^{\text {a }}$, I. Slaus ${ }^{\text {b }}$, B. Vuaridel ${ }^{\text {, }}$, \\ F. Sperisen ${ }^{d}$, W. Kretschmer ${ }^{\mathrm{e}}$, A. Rauscher ${ }^{\mathrm{e}}$, W. Schuster ${ }^{\mathrm{e}}$, R. Weidmann ${ }^{\mathrm{e}}$, M. Haller ${ }^{\mathrm{e}}$, \\ M. Bruno ${ }^{f}$, F. Cannata ${ }^{f}$, M. D'Agostino ${ }^{f}$, H. Witała ${ }^{g}$, Th. Cornelius ${ }^{g}$, W. Glöckle ${ }^{g}$ \\ and P.A. Schmelzbach ${ }^{\mathrm{h}}$ \\ a Institute for Intermediate Energy Physics, ETH, CH-8093 Zürich, Switzerland \\ b Ruder Boskovic Institute, YU-41000 Zagreb, Yugoslavia \\ c University of Michigan, Ann Arbor, MI 48109, USA \\ and BNL, Upton, NY 11973, USA \\ d IUCF, Bloomington, IN 47405, USA \\ e University of Erlangen, D-8520 Erlangen, FRG \\ ' INFN, I-40126 Bologna, Italy \\ g Ruhr University Bochum, D-4630 Bochum 1, FRG \\ ${ }^{\mathrm{h}}$ Paul Scherrer Institute (PSI), CH-5232 Villigen, Switzerland
}

Received 1 May 1990

\begin{abstract}
Proton-deuteron elastic scattering has been investigated at $E_{\mathrm{p}}=22.7 \mathrm{MeV}$ by comparison of rigorous Faddeev calculations with experimental results. The observable most sensitive to the tensor force is the nucleon-nucleon polarization transfer coefficient $K_{y}^{y^{\prime}}$. The new angular distribution of $K_{y}^{y^{\prime}}$ clearly favours the tensor force of the Bonn A potential, which is weaker than the one of the Paris potential.
\end{abstract}

The determination of the nucleon-nucleon $(\mathrm{N}-\mathrm{N})$ force is still the fundamental problem in nuclear physics. Several realistic N-N potentials based on meson theory are now available; e.g. Paris [1], Bonn [2,3] and Nijmegen [4]. The Bonn potential of ref. [2], (Bonn OBEPQ(A), called Bonn A in the following) has a significantly weaker tensor force than the Paris and Nijmegen potentials and the Bonn OBEPQ(B) potential of ref. [3], called Bonn B. The weaker tensor force in the Bonn A potential is associated to a larger central force, which provides a larger triton binding energy [5]. The notorious discrepancy between the theoretical and experimental binding energy of about $1 \mathrm{MeV}$ is thus reduced and only a few hundred $\mathrm{keV}$ discrepancy are left to be accounted for by three nucleon force ( $3 \mathrm{NF}$ ) effects. A legitimate question is which of the established potentials is closer to reality and what is the strength of the tensor force. Unfortunately, the neutron-proton scattering data obtained so far do not determine the ${ }^{3} \mathrm{~S}_{1}-{ }^{3} \mathrm{D}_{1}$ mixing parameter $\varepsilon_{1}$ with sufficient accuracy, because at low energy in general only differential cross section and analyzing power $A_{y}$ data are available. The values of $A_{y}$ are very small, i.e. only about $1 \%$, and because of the difficulties of experiments induced by neutrons the relative accuracy is low compared to $\mathrm{p}-\mathrm{p}$ scattering data. The values of $\varepsilon_{1}$ found in analyses of experimental results [6] are displayed in fig. 1 together with the potential predictions. Also recent measurements of the neutron-proton spin correction parameter $A_{y}$ below $50 \mathrm{MeV}$ [7], which gave new values for $\varepsilon_{1}$, still cannot distinguish between the three curves in fig. 1. At $325 \mathrm{MeV}$, where experimental polarization transfer coefficients $K_{y}^{y^{\prime}}$ and $K_{x}^{x^{\prime}}$ are available, the uncertainties in $\varepsilon_{1}$ range between $1^{\circ}$ and $7^{\circ}$ [8]. In summary the present $n-p$ data set does not allow to pin down the strength of the ${ }^{3} \mathrm{~S}_{1}-{ }^{3} \mathrm{D}_{1} \mathrm{~N}-\mathrm{N}$ force. On the other hand that $\mathrm{N}-\mathrm{N}$ force component is the most decisive one for nuclear binding in general.

The spin observables in the nucleon-deuteron $(\mathrm{N}-$ D) system are much larger than those in the $\mathrm{N}-\mathrm{N}$ system and their measurements can give additional 


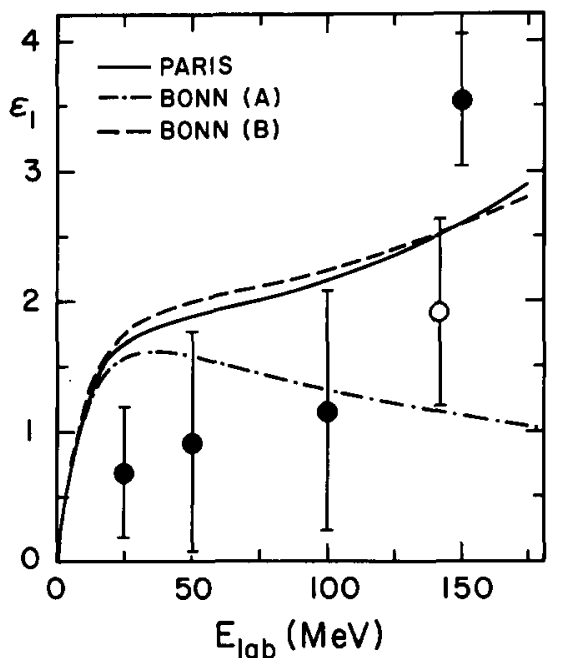

Fig. 1. The $N-N{ }^{3} S_{1}-{ }^{3} D_{1}$ mixing parameter $\varepsilon_{1}$ as given by Arndt's phase-shift analysis (dots) [6] and by the Paris, Bonn $A$ and Bonn B potentials. The open circle is from Dubois et al., ref. [6].

information on $\mathrm{N}-\mathrm{N}$ force properties provided that a rigorous three body theory is employed. Rigorous means that given a $3 \mathrm{~N}$ hamiltonian the Schrödinger equation can be solved in a numerically precise manner and agreement or disagreement with $3 \mathrm{~N}$ data unambiguously reflects the underlying dynamics and is not plagued by uncontrolled approximations. In the present study we work with $\mathrm{N}-\mathrm{N}$ forces only. Clearly it is conceivable that in a $3 \mathrm{~N}$ system the $\mathrm{N}-\mathrm{N}$ forces may change in the presence of a third nucleon and proper $3 \mathrm{NF}$ will act in addition to $\mathrm{N}-\mathrm{N}$ forces. Then without knowledge of those effects and sticking to a dynamical picture of pure $\mathrm{N}-\mathrm{N}$ forces the analysis of the $3 \mathrm{~N}$ system may lead to $\mathrm{N}-\mathrm{N}$ force properties, which may mock up neglected dynamics. If the $\mathrm{N}-\mathrm{N}$ force properties so determined, however, are fully compatible with $\mathrm{N}-\mathrm{N}$ data, those $\mathrm{N}-\mathrm{N}$ forces are as legitimate as the ones which are fitted to $\mathrm{N}-\mathrm{N}$ data only. It simply reflects the fact that the $\mathrm{N}-\mathrm{N}$ data available up to now are not precise and sensitive enough to pin down the $\mathrm{N}-\mathrm{N}$ forces. It is in this spirit that we understand the present study. Only future theoretical insight into nuclear dynamics will tell finally which portion of a $3 \mathrm{~N}$ observable is caused by effects beyond the free $\mathrm{N}-\mathrm{N}$ forces. Rigorous $3 \mathrm{~N}$ scattering calculations using free $\mathrm{N}-\mathrm{N}$ interactions from the Paris and Bonn potentials and allowing for the charge dependence of the $\mathrm{N}-\mathrm{N}$ force are now available [9]. They are superior to those using phenomenological separable $\mathrm{N}-\mathrm{N}$ potentials of different rank and different type of form factors. The Bonn potential is based on the $n-p$ system and has consequently a stronger ${ }^{1} S_{0}$ force than the Paris potential, which is adjusted to the p-p system. In the results presented here we take that fact into account by using the following effective two-body $t$-matrix in the state ${ }^{1} \mathrm{~S}_{0}: t=\frac{2}{3} t_{\mathrm{np}}+\frac{1}{3} t_{\mathrm{nn}}$. This is the correct linear combination in the state of total isospin $T=\frac{1}{2}$ [10].

We found that from the tensor analyzing powers only $T_{22}$ shows some sensitivity to the details of the tensor force and depends less on higher partial wave force components than $T_{21}$ and $T_{20}$. Also in $\mathrm{p}-\mathrm{d}$ scattering at $10 \mathrm{MeV}$ the $T_{22}$ data [11] and the nucleon to nucleon polarization transfer coefficients $K_{y}^{y^{\prime}}$ and $K_{z}^{x^{\prime}}$ showed some sensitivity to the tensor force [12]. The comparison of the rigorous calculation with the $K_{y}^{y^{\prime}}$ and $K_{z}^{x^{\prime}}$ data $[11,13]$ was not conclusive [12], since the predictions with the $j \leqslant 3$ force components gave about an equally good overall agreement from the Paris and Bonn A potentials.

In any case the sensitivities found made it promising to study these observables again at higher energies, where as a bonus Coulomb effects, which are neglected in the calculations, should be smaller. The present rigorous calculations perfectly agree with the data at $22.7 \mathrm{MeV}$ for the differential p-d cross section for $\theta_{\mathrm{cm}} \geqslant 40^{\circ}$ and the tensor analyzing powers $T_{20}$ and $T_{22}$. We show in fig. 2 the tensor analyzing power $T_{20}$ at $22.7 \mathrm{MeV}$ [14] compared to our rigorous calculations with the Paris, Bonn A and Bonn B potentials. The very good agreement suggests that at this energy Coulomb force effects are not relevant. The near coincidence of the Bonn A, Bonn B and Paris potential predictions also indicates that the tensor analyzing powers at this energy are not particularly sensitive to the details of the tensor force. Furthermore, fig. 2 shows that in contrast to $A_{y}$ the ST calculation ( ${ }^{1} \mathrm{~S}_{0}$ and ${ }^{3} \mathrm{~S}_{1}-{ }^{3} \mathrm{D}_{1} \mathrm{~N}-\mathrm{N}$ force components only) produces already essentially the shape of the observable. Also the strong differences between the tensor forces in Bonn $\mathrm{A}$ and Paris potentials are hardly visible. The inclusion of higher partial wave force components moves the theoretical predictions into the range of the overall experimental errors. Very much the same picture is true for $T_{22}$. Therefore, we 


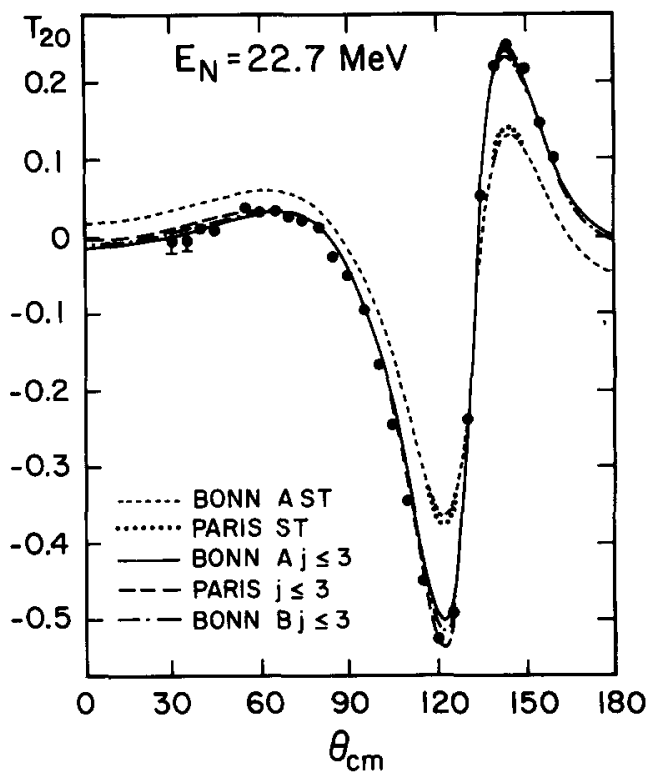

Fig. 2. The p-d tensor analyzing power $T_{20}$ at $22.7 \mathrm{MeV}$ from ref. [14] compared to rigorous solutions of the Faddeev equations including the Paris, Bonn $\mathrm{A}$ and Bonn $\mathrm{B} \mathrm{N}-\mathrm{N}$ potentials for the ST states and for all states with total N-N angular momenta $j \leqslant 3$. ST means ${ }^{1} S_{0}+\left({ }^{3} S_{1}-{ }^{3} D_{1}\right)$ forces.

conclude that these tensor analyzing powers are not sensitive to $\varepsilon_{1}$.

In a search for observables sensitive to the tensor force we performed a new measurement of $K_{y}^{y^{\prime}}(\theta)$ at an incident proton energy of $22.7 \mathrm{MeV}$. This second order polarization observable is determined by a double scattering experiment. In the first scattering by an angle $\theta$, with an analyzing power $A_{y}(\theta)$, the polarization $p_{1}$ of the beam is changed to $p_{2}$ according to

$p_{2}(\theta)=\frac{A_{y}(\theta)+K_{y}^{y^{\prime}}(\theta) \cdot p_{1}}{1+A_{y}(\theta) \cdot p_{1}}$.

The $22.7 \mathrm{MeV}$ polarized proton beam from the PSI cyclotron was scattered from a deuterium target which was pressurized to 16 bar and cooled to $77 \mathrm{~K}$. The scattered protons were focused by a magnetic quadrupole triplet lens into the polarimeters $2 \mathrm{~m}$ from the first scattering chamber. The beam polarization $p_{1}$ was continuously monitored by a ${ }^{12} \mathrm{C}$ polarimeter located upstream of the deuterium target. The incident beam polarization was also calibrated by replacing the $\mathrm{D}_{2}$ target with a ${ }^{4} \mathrm{He}$ target and comparing our measurement with the calibration from ref. [15]. The sign of the beam polarization was inverted every few seconds. This method allowed the determination of $K_{y}^{y^{\prime}}$ from the ratios of the detector counting rates independently of solid angles. For the determination of $K_{y}^{y^{\prime}}$ one also needs the values of the vector analyzing powers $A_{4}(\theta)$. For this aim the values from ref. [14] were used.

One of the main experimental problems is the measurement of the polarization $p_{2}$ of the scattered protons over a large angular range, since their energy decreases rapidly with scattering angle. Two different polarimeters based on $p-\alpha$ and $p-{ }^{12} \mathrm{C}$ scattering were used to cover the energies of the protons scattered from $\theta_{\mathrm{cm}}=45^{\circ}$ to $125^{\circ}$. Details of these polarimeters, their calibrations and their use in polarization transfer experiments for the same energy of protons incident into the polarimeters are given in refs. $[11,16]$. The background observed in the present experiment is even smaller since an additional $\Delta E$ detector in front of the polarimeter was used in coincidence with the side detectors. As a stringent consistency test the polarization transfer coefficient $K_{y}^{y^{\prime}}$ in the sensitive region has been measured using both the ${ }^{4} \mathrm{He}$ polarimeter and the ${ }^{12} \mathrm{C}$ polarimeter. The two values agree very well within the error of the individual results of \pm 0.02 .

The measured and calculated $K_{y}^{y^{\prime}}$ are shown in fig. 3a. The present data clearly favour the Bonn A potential with the weaker tensor force. Bonn $B$ and Paris potential predictions are close together in accordance with their $\varepsilon_{1}$ parameter shown in fig. 1 . In fig. $3 \mathrm{~b}$ the sensitivity of $K_{y}^{y^{\prime}}$ to the addition of $\mathrm{N}-\mathrm{N}$ force components is demonstrated. Again, the ST forces alone provide the basic shape, however, there is a clear distinction between Bonn A and Paris potential predictions. Since we use the $\frac{2}{3}-\frac{1}{3}$ rule for the ${ }^{1} S_{0}$ state and therefore the ${ }^{1} S_{0}$ force is the same for both calculations the reason lies totally in the different ${ }^{3} \mathrm{~S}_{1}-{ }^{3} \mathrm{D}_{1}$ force components. Inclusion of all $j \leqslant 2$ force components for Bonn A and Paris potentials keeps the two curves still apart and the effect of the different ${ }^{3} S_{1}-{ }^{3} D_{1}$ force remains. Finally the inclusion of the $j=3$ force components leads to the curves shown in fig. 3a. Though the inclusion of $p$ - and $d$-wave forces is noticeable, the sensitivity to changes in these forces is weak as we found by various studies interchanging 

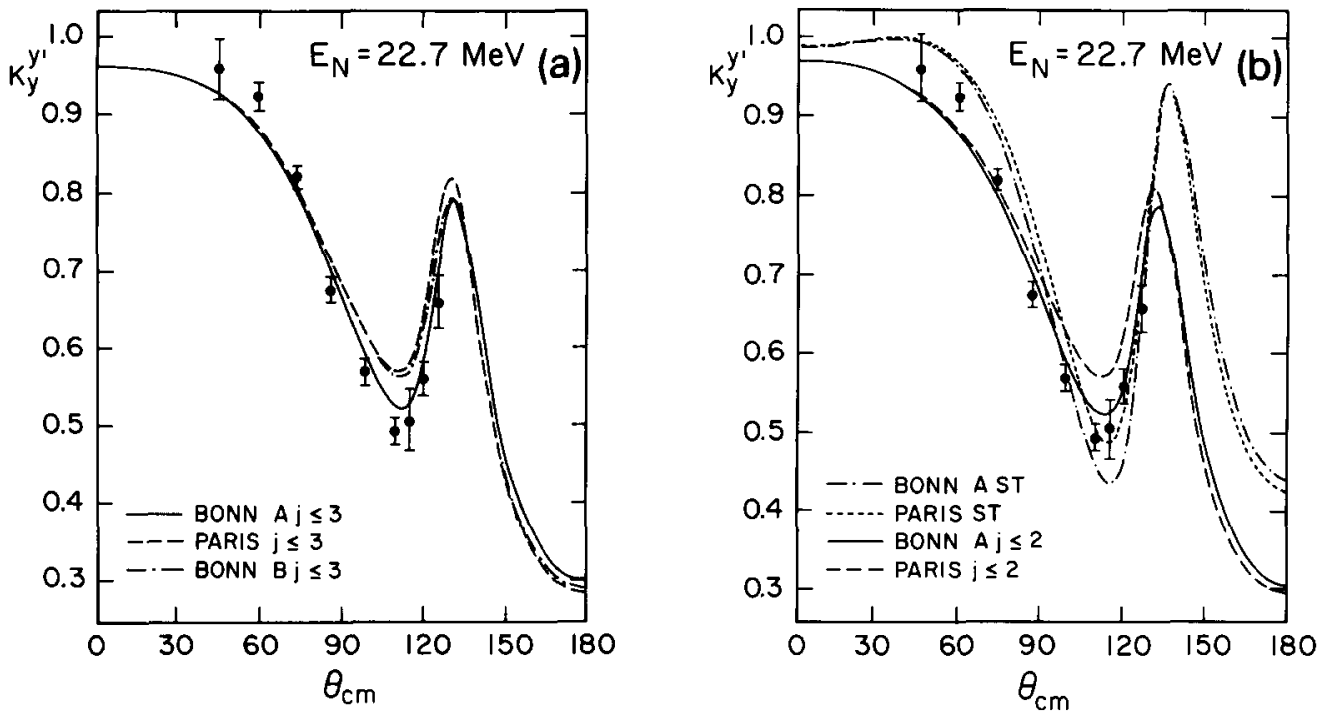

Fig. 3. (a) The experimental nucleon spin transfer coefficient $K_{y}^{y^{\prime}}$ at $22.7 \mathrm{MeV}$ compared to rigorous solutions of the Faddeev equations for the Paris, Bonn A and Bonn B N-N potentials in all states $j \leqslant 3$. (b) The nucleon spin transfer coefficient $K_{y}^{y^{\prime}}$ at 22.7 MeV for the Paris and Bonn A potentials for the ST and $j \leqslant 2$ states. ST means ${ }^{1} \mathrm{~S}_{0}+\left({ }^{3} \mathrm{~S}_{i}-{ }^{3} \mathrm{D}_{1}\right)$ forces.

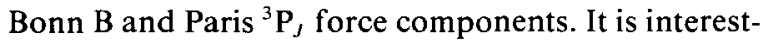
ing to note that $K_{y}^{y^{\prime}}$ is sensitive also to the ${ }^{1} \mathrm{~S}_{0}$ force. For instance using the Bonn A potential also in the ${ }^{1} S_{0}$ state instead of allowing charge dependence, the Bonn A curve is shifted downwards right into the experimental values. This however would not be an acceptable procedure since the ${ }^{1} \mathrm{~S}_{0} \mathrm{~N}-\mathrm{N}$ phases are very well determined and the charge dependence has to be taken into account. The remaining discrepancy in fig. 3a may be calling for an even weaker tensor force than in Bonn A.

We know from a comparison of our rigorous calculation to neutron-deuteron and proton-deuteron analyzing power data at 10 and $22.7 \mathrm{MeV}[12,17]$ that the ${ }^{3} \mathrm{P}_{J}$ phase shifts of the Bonn or Paris potentials have to be modified substantially to remove the discrepancy with the vector analyzing power data. This may include charge-independence breaking in ${ }^{3} \mathrm{P}$ waves. We stress that drastic changes in the ${ }^{3} \mathrm{P}_{J}$ forces did not lead to a noticeable shift of the theoretical curve of $K_{y}^{y^{\prime}}$.

The systematic investigation of the sensitivity of all proton-deuteron elastic scattering observables at 22.7 MeV to the tensor force leads to the conclusion that the most promising observable is the nucleon to nucleon polarization transfer coefficient $K_{y}^{y^{\prime}}$ and to a smaller extent the nucleon to deuteron transfer coefficients $K_{y}^{y^{\prime}}$ and $K_{z}^{x^{\prime}}$.

The important issue is now whether the apparent preference for the weaker tensor force can be influenced by small uncertainties in our study. Since the systematic uncertainties in the present measurement (background subtraction, polarization of the incident beam, $A_{y}$ values of p-d scattering and the calibration of the polarimeter) are smaller than 0.02 , the data in the angular region $90^{\circ} \leqslant \theta_{\mathrm{cm}} \leqslant 125^{\circ}$ still unambiguously favour the weaker tensor force. The calculations neglect the Coulomb force and there are no rigorous estimates about its effects. A model calculation [18] (strong forces of rank one only) including the Coulomb force in the approximation that the two-body off-shell Coulomb $t$-matrix is replaced by the Coulomb potential, which was performed at the very low energy of $E_{\text {plab }}=2.5 \mathrm{MeV}$, leads to small Coulomb effects in the differential cross section and tensor analyzing powers. First order approximations to include Coulomb force effects at $10 \mathrm{MeV}$ [11] lead to only insignificant changes in $K_{y}^{y^{\prime}}$. The fact that the calculations reproduce well the p-d data at 10 and 22.7 MeV makes it very unlikely that Coulomb effects are important and moreover would have conspired just to lower the minimum in $K_{y}^{v^{\prime}}$ around $90^{\circ}-120^{\circ}$. 
Our theoretical analysis is based on pure $\mathrm{N}-\mathrm{N}$ forces. The present data clearly favour the predictions of the rigorous three-body calculation using the Bonn A potential. Though three-nucleon data sometimes provide a better insight into the $\mathrm{N}-\mathrm{N}$ interaction than the two nucleon data, because they turn out to be more sensitive, our data considered alone cannot yet decisively prove in favour of a weaker tensor force. Namely, albeit all on-shell N-N interactions relevant for the investigated observables are fully under control, our calculation does not include the Coulomb force and the three-nucleon force. The inclusion of both of them might take quite some time, and the $3 \mathrm{NF}$ effects in the three nucleon system are not adequately understood, even not for the bound state problem. Comparison between our calculations using Bonn A, Bonn B and Paris potentials indicates that the off-shell effects and consequently the three nucleon force effects, i.e. the differences between Paris and Bonn B predictions, are considerably smaller than the effects due to the tensor force difference, which is the difference between Bonn A and Bonn B potentials. Therefore, taken together with information on the three nucleon bound state and on nuclear matter, our results argue in favour of the weaker tensor force.

This work was supported by the Swiss National Science Foundation, the Paul Scherrer Institute and the Bundesministerium für Forschung und Technologie (FRG, contract number 06ER185).

\section{References}

[1] M. Lacombe et al., Phys. Rev. 21 (1980) 861.

[2] R. Machleidt, K. Holinde and Ch. Elster, Phys. Rep. 149 (1987) 1.

[3] R. Machleidt, Adv. Nucl. Phys. 19 (1989) 189.

[4] M.M. Nagels, T.A. Rijken and J.J. de Swart, Phys. Rev. D 17 (1978) 768 .

[5] R.A. Brandenburg et al., Phys. Rev. C 37 (1988) 781; LANL Report No. LA-UR 86-3700 (1986), unpublished; T. Sasakawa, Nucl. Phys. A 463 (1987) 327C.

[6] R.A. Arndt, J.S. Hyslop III and L.D. Roper, Phys. Rev. D 35 (1987) 128;

R. Dubois et al., Nucl. Phys. A 377 (1982) 554.

[7] P. Doll et al., Contributed paper Intern. Few Body XII Conf. (Vancouver, 1989), TRIUMF report TRI-89-2, p. C16.

[8] G.S. Chulick et al., Phys. Rev. C 37 (1988) 1549.

[9] H. Witała, T. Cornelius and W. Glöckle, Few Body Systems 3 (1988) 123; Nucl. Phys. A 491 (1989) 157.

[10] H. Witała, W. Glöckle and T. Cornelius, Phys. Rev. C 39 (1989) 384.

[11] F. Sperisen et al., Nucl. Phys. A 422 (1984) 81.

[12] H. Witała, T. Cornelius and W. Glöckle, Nucl. Phys. A 496 (1989) 446.

[13] F. Sperisen et al., Phys. Lett. B 102 (1981) 9.

[14] W. Grüebler et al., Nucl. Phys. A 398 (1983) 445.

[15] A.D. Bacher et al., Phys. Rev. C 5 (1972) 1147.

[16] F. Sperisen et al., Nucl. Instrum. Methods 190 (1981) 301; 204 (1983) 491.

[17] H. Witała, W. Glöckle and T. Cornelius, in preparation.

[18] G.H. Berthold, A. Stadler and H. Zankel, Phys. Rev. Lett. $61(1988) 1077$ 